\title{
Clarificación y Epitipificación de Tagetes iltisiana (Asteraceae, Tageteae)
}

\author{
Clarification and Epitypification of Tagetes iltisiana (Asteraceae, \\ TAgeteae)
}

\author{
Dario J. Schiavinato ${ }^{1,2, *}$ ) y Adriana Bartoli1 ${ }^{1}$
}

1. Cátedra de Botánica Sistemática, Facultad de Agronomía, Universidad de Buenos Aires, Av. San Martín 4453, 1417, Buenos Aires, Argentina

2. Consejo Nacional de Investigaciones Científicas y Técnicas (CONICET)

*schiavi@agro.uba.ar

\section{Citar este artículo}

SCHIAVINATO, D. J. \& A. BARTOLI. 2019. Clarificación y Epitipificación de Tagetes iltisiana (Asteraceae, Tageteae). Bol. Soc. Argent. Bot. 54: 589-598.

DOI: http://dx.doi. org/10.31055/1851.2372.v54. n4.24954

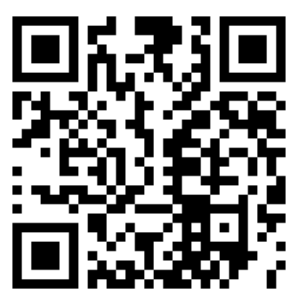

Recibido: 15 Julio 2019

Aceptado: 23 Septiembre 2019

Publicado: 15 Diciembre 2019

Editor: Franco Ezequiel Chiarini (D)

\section{SUMMARY}

Background and aims: Tagetes is an American genus that includes $50-55$ species of annual and perennial, strongly aromatic herbs. As part of the integral revision of the genus, in this work we study morphology and distribution of $T$. iltisiana, an annual Bolivian species with a dubious taxonomic position, occasionally included in the synonymy of Tagetes laxa, an annual species that inhabits northwestern Argentina.

M\&M: We analyzed common herbarium specimens and we conducted field observations in Jujuy, Salta and Tucumán provinces, keeping reference vouchers at Gaspar Xuarez Herbarium (BAA). Then, we compared these materials with the protologues and type specimens of Tagetes iltisiana y Tagetes laxa.

Results: Numerous important morphological characters were detected to differentiate both entities. An epitype of Tagetes iltisiana is designated. A revised morphological description of Tagetes iltisiana is presented. An illustration of Tagetes iltisiana is presented for the first time. A key to differentiate the annual species of Tagetes that inhabit north-west Argentina and Bolivia is provided.

Conclusions: The synonymy of Tagetes iltisiana within Tagetes laxa is rejected, identifying them as two different species. The presence of Tagetes iltisiana in Argentina is reported for the first time, bringing the number of Argentinian species of this genus to 15 .

\section{KEY WORDS}

Asteraceae, epitype, Tagetes, taxonomy.

\section{RESUMEN}

Introducción y objetivos: Tagetes es un género americano que incluye 50-55 especies de hierbas anuales o perennes, fuertemente aromáticas. Como parte de la revisión integral del género, en el presente trabajo se estudió la morfología y distribución de Tagetes iltisiana, una especie boliviana anual muy poco conocida y con una posición taxonómica dudosa, ubicada ocasionalmente bajo la sinonimia de Tagetes laxa, una especie anual que habita en el noroeste argentino.

M\&M: Se analizaron ejemplares comunes de herbario y se realizaron observaciones a campo en las provincias de Jujuy, Salta y Tucumán, conservándose ejemplares de referencia en el herbario Gaspar Xuarez (BAA). Estos materiales fueron posteriormente comparados con los materiales originales y protólogos de Tagetes iltisiana y Tagetes laxa.

Resultados: Se detectaron numerosos caracteres diagnóstico de importancia para diferenciar ambas entidades. Se designa un epitipo para Tagetes iltisiana, se presenta una descripción morfológica ampliada para esta especie y se presenta una ilustración por primera vez. Se presenta una clave para diferenciar las especies anuales de Tagetes que crecen en el Noroeste Argentino y Bolivia.

Conclusiones: Se rechaza la sinonimia de Tagetes iltisiana con Tagetes laxa, identificándolas como dos entidades diferentes. Se registra por primera vez la presencia de Tagetes iltisiana en Argentina, elevando el número de especies de este género a 15 en dicho país.

Palabras clave

Asteraceae, epitipo, Tagetes, taxonomía. 


\section{INTRODUCCIÓN}

Tagetes L. (1753: 887) incluye ca. 50-55 especies distribuidas desde el suroeste de los Estados Unidos hasta el centro de Chile y el norte de la Patagonia Argentina (Neher, 1966; Schiavinato et al. 2017). El género comprende plantas anuales o perennes, fuertemente arómaticas, siendo sus principales características la presencia de hojas con láminas generalmente pinnatisectas, involucro de filarios fusionados en una sola serie y papus conformado por aristas o escamas de diversos tamaños y formas (Schiavinato \& Bartoli, 2018).

Tagetes laxa Cabrera (1937: 189) fue descripta originalmente como una hierba anual para la provincia de Tucumán, Argentina. Posteriormente, su distribución fue ampliada a las provincias de Jujuy, Salta y Catamarca, en laderas húmedas entre los 2000-4000 m (Cabrera, 1978; Ariza Espinar, 1997; Gutierrez \& Stampacchio, 2015).

Tagetes iltisiana H.Rob. (1973: 378) fue descripta como una hierba anual para el departamento de Cochabamba, Bolivia. El holotipo de T. iltisiana fue coleccionado en 1968, y las colecciones posteriores fueron muy escasas. En su tesis doctoral, Soule (1993) incluyó a T. iltisiana en la sinonimia de $T$. laxa, pero dicho trabajo nunca fue válidamente publicado. La misma autora colocó en 1995 un determinavit en el holotipo de T. iltisiana, identificándolo como T. laxa.

Hasta la fecha, no hay ningún estudio taxonómico que incluya ambas especies. Los últimos tratamientos del género Tagetes para Argentina (Ariza Espinar, 1997; Gutierrez \& Stampacchio, 2015) no incluyen a T. iltisiana. Por otro lado, en un listado preliminar de plantas vasculares para Bolivia, Hind (2011) reconoció provisoriamente a T. iltisiana como una especie válida pero con dudas sobre su sinonimia con T. laxa. Los últimos listados de plantas bolivianas (Robinson, 2014; Ulloa Ulloa et al., 2017) reconocen a ambas como especies de la flora de dicho país.

Como parte de la revisión (en progreso) de las especies sudamericanas de Tagetes, se hallaron ejemplares de herbario coleccionados en el noroeste Argentino y determinados bajo T. laxa que no parecían estar asociados a la descripción original ni al material tipo de este taxón. Esto motivó un viaje de colección a las provincias de Jujuy, Salta y Tucumán, con el propósito de estudiar estos individuos en su hábitat natural. Las observaciones a campo permitieron corroborar que estos ejemplares correspondían a una entidad diferente de T. laxa. El estudio del protólogo y material original de Tagetes iltisiana y el posterior cruzamiento de la información obtenida con los ejemplares hallados a campo permitieron concluir que los mismos correspondían a este nombre, rechazando así la sinonimia de $T$. iltisiana con T. laxa.

En el presente trabajo, se amplía la descripción de Tagetes iltisiana y se designa un epitipo para dicho nombre. Se amplía la distribución de $T$. iltisiana, incorporando a esta especie a la Flora Argentina. Asimismo, se presenta por primera vez una ilustración para esta especie. Finalmente, se provee una clave dicotómica para diferenciar esta especie de las demás especies anuales de Tagetes que habitan Bolivia y el noroeste Argentino: $T$. filifolia Lag. (1816: 28), T. imbricata Schiavinato \& Adr.Bartoli (2019: 277), T. laxa, T. minuta L. (1753: 887), T. multiflora Kunth (1818: 154), T. praetermissa (Strother 1968: 343) H.Rob. (1973: 380), y T. terniflora Kunth (1818: 154).

\section{Materiales y Métodos}

Se analizaron especímenes comunes de herbario conservados en las siguientes instituciones: BAA, CORD, LIL, LP, MCNS, SI y US (Thiers, 2019). Se realizaron observaciones a campo en las provincias de Jujuy, Salta y Tucumán, conservándose ejemplares de referencia en el herbario Gaspar Xuarez (BAA). Se analizaron los protólogos y ejemplares tipo de Tagetes iltisiana y Tagetes laxa.

Para la descripción de T. iltisiana se emplearon los siguientes caracteres morfológicos: hábito, hojas, capítulos, filarios, flores y aquenios.

\section{Resultados y Discusión}

\section{Delimitación de Tagetes laxa}

El análisis morfológico de ejemplares comunes de herbario, determinados previamente bajo Tagetes laxa, permitió reconocer dos entidades claramente delimitadas. Si bien los ejemplares secos pueden presentar similitudes en cuanto a su aspecto general (plantas anuales con hojas pinnatisectas, con capitulescencias corimbiformes laxas y papus 


\section{J. Schiavinato y A. Bartoli - Clarificación y Epitipificación de Tagetes iltisiana}

formado por pocos elementos paleáceos), un estudio detallado de hojas, involucros y flores permitió postular la existencia de dos entidades bien definidas: una de ellas comprendía ejemplares con hojas concoloras, involucros 5-7-lobados y corola de las flores marginales con limbo oblongo $\mathrm{u}$ orbicular, mientras que la otra se caracterizaba por presentar ejemplares con hojas discoloras, involucros 3-lobados (raramente 4-lobados) y corola de las flores marginales con limbo oblato. Estos caracteres morfológicos fueron posteriormente observados a campo, confirmando su valor taxonómico para diferenciar ambas entidades.

El estudio del protólogo y material original de $T$. laxa, y su posterior comparación con los ejemplares comunes de herbario y los especímenes hallados a campo, reveló que la primera entidad delimitada (hojas concoloras, involucros 5-7-lobados y limbo oblongo u orbicular) se incluía inequívocamente dentro de la circunscripción del mencionado taxón (Fig. 1). Por el contrario, la segunda entidad definida a partir de la observación del material coleccionado (hojas discoloras, involucros 3-lobados y limbo oblato) no parecía estar asociada a $T$. laxa ni a ninguna de las demás especies del género previamente citadas para la Flora Argentina.

\section{Clarificación de Tagetes iltisiana}

Al continuar con el estudio de las especies de Tagetes descriptas para Bolivia, se hallaron
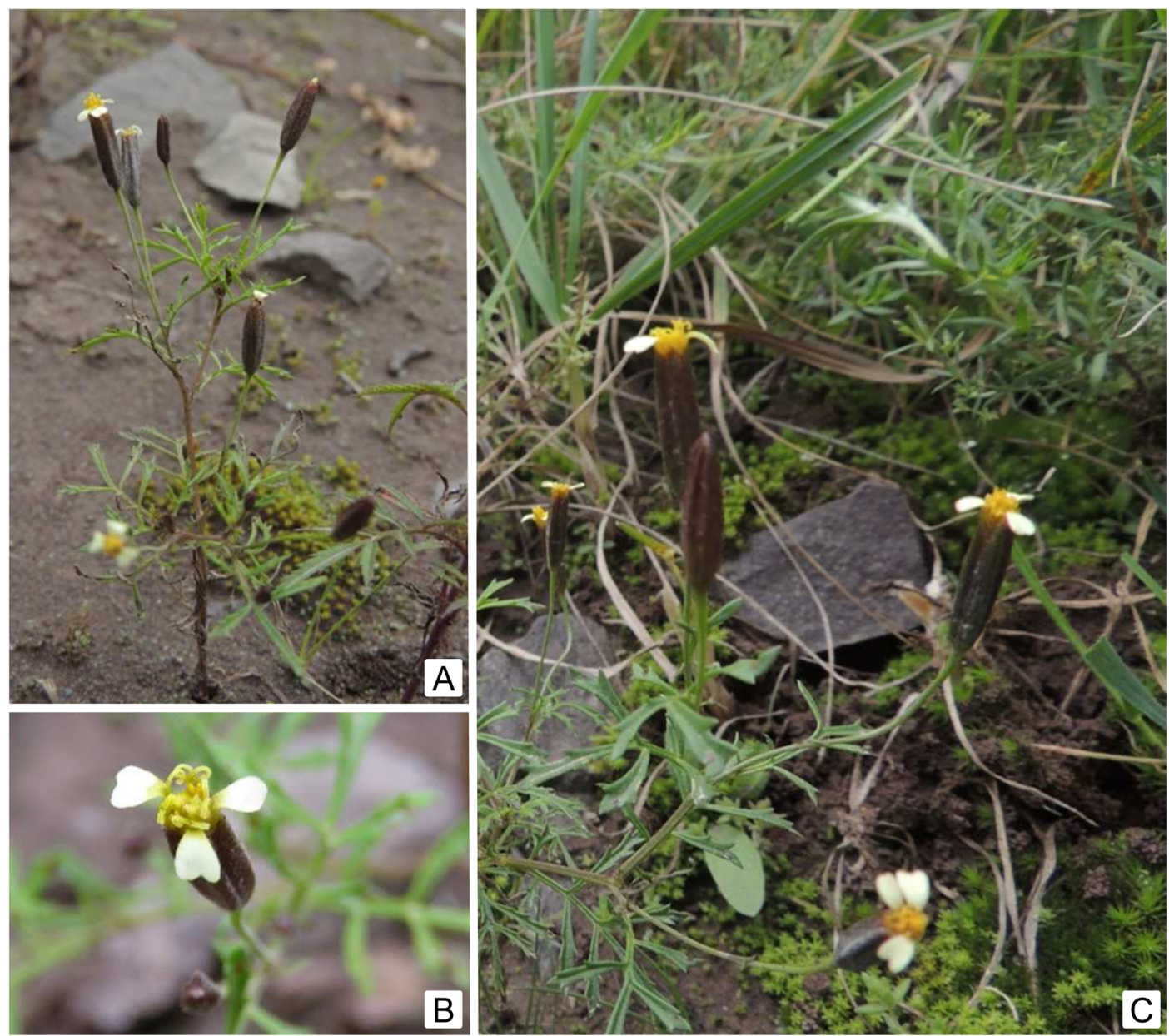

Fig. 1. Tagetes laxa. A: Planta. B-C: Capítulos. 
coincidencias entre el material examinado y la descripción original de Tagetes iltisiana, en la cual se destaca el involucro 3-lobado como un carácter de valor para distinguir dicho taxón de sus congéneres. Sin embargo, algunos caracteres mencionados en la descripción original diferían notablemente de lo observado a campo. Ante la falta de un tratamiento taxonómico previo que incluya a $T$. iltisiana, se realizó un estudio en detalle del protólogo y del material original, para confirmar si la segunda entidad mencionada correspondía a la circunscripción de este taxón y, en caso de ser afirmativo, poder delimitarlo correctamente.

La observación del material original de $T$. iltisiana, integrado solamente por el holotipo, reveló la existencia de caracteres que no fueron incluidos en su descripción original. Uno de ellos es la presencia de hojas discoloras, que también fue observado en la segunda entidad hallada a campo. Otros caracteres no mencionados en el protólogo que pudieron ser observados en el holotipo son la presencia de hojas alternas en la parte superior de las plantas y la presencia de tricomas cortos y escasos en la epidermis abaxial de algunas hojas.

Por otro lado, se detectaron algunos caracteres incluidos en la descripción original de T. iltisiana que no coincidían con el material original. Uno de ellos es el papus, que en el protólogo es descripto como 2-3-seriado, con elementos paleáceos escamiformes en la serie externa y elementos paleáceos aristiformes en la serie interna. La observación detallada de los aquenios hallados en el holotipo permitió verificar que, si bien los elementos paleáceos escamiformes parecen ubicarse hacia el exterior, éstos se originan realmente entre las bases de los elementos aristiformes, por lo que estructuralmente todos los elementos del papus se disponen en una única serie.

Finalmente, se detectaron caracteres mencionados en la descripción original que no pudieron ser contrastados en el holotipo debido a la insuficiencia de este material. Uno de ellos es el tamaño y forma del limbo de la corola de las flores marginales, que en el protólogo es descripto como orbicular y de $1 \mathrm{~mm}$ de diámetro, pero que no pudo ser observado en el holotipo debido a que el mismo no contaba con ninguna flor marginal completa. Una posible explicación para esta diferencia observada entre el protólogo de T. iltisiana y el material estudiado en herbarios y observado a campo, es que la descripción original pudo haber sido realizada a partir de una flor marginal destruida o incompleta.

La comparación entre el holotipo y protólogo de T. iltisiana y el material común examinado, con las coincidencias halladas, permite postular que estos especímenes corresponden a una misma entidad. La misma, como se ha mencionado, puede ser diferenciada de $T$. laxa (Tabla 1) y de otras especies anuales de Tagetes que habitan la región en estudio, por lo que se concluye que la segunda entidad observada corresponde a la circunscripción de T. iltisiana (Fig. 2). Este hallazgo representa una nueva cita para la Flora Argentina, lo cual asciende el número de especies de Tagetes presentes en dicho país a 15 .

\section{Epitipificación de Tagetes iltisiana}

El holotipo de Tagetes iltisiana se encuentra depositado en US (US 00124917, imagen disponible en $<$ http://n2t.net/ark:/65665/308e3c7a9-bbfc401a-817f-943784800527>). Este espécimen consta de un único individuo que presenta hojas deterioradas o incompletas y pocos capítulos, de los cuales solamente uno se encuentra abierto, con aquenios maduros y ninguna flor completa. Esta escasez de material vegetativo y reproductivo dificulta notoriamente la correcta identificación y caracterización de la especie, ya que no pueden apreciarse en este espécimen los caracteres diagnóstico mencionados en el apartado anterior. En el protólogo, Robinson (1973) menciona un isotipo en WIS, pero dicho espécimen nunca fue hallado

\begin{tabular}{|c|c|c|}
\hline Carácter & T. laxa & T. iltisiana \\
\hline $\begin{array}{l}\text { Coloración de } \\
\text { las hojas }\end{array}$ & $\begin{array}{c}\text { Concoloras, } \\
\text { verdes en } \\
\text { ambas } \\
\text { superficies }\end{array}$ & $\begin{array}{c}\text { Discoloras, } \\
\text { verde claro en } \\
\text { epidermis abaxial } \\
\text { y verde oscuro en } \\
\text { epidermis adaxial }\end{array}$ \\
\hline $\begin{array}{c}\text { Número de filarios } \\
\text { por involucro }\end{array}$ & $5-7$ & $3(-4)$ \\
\hline $\begin{array}{l}\text { Forma y ancho } \\
\text { del limbo de las } \\
\text { flores marginales }\end{array}$ & $\begin{array}{l}\text { Oblongo- } \\
\text { orbicular, } \\
\text { 0,6-1,3 mm } \\
\text { de ancho }\end{array}$ & $\begin{array}{l}\text { Oblato, } 2,5-3,0 \\
m m \text { de ancho }\end{array}$ \\
\hline
\end{tabular}




\section{J. Schiavinato y A. Bartoli - Clarificación y Epitipificación de Tagetes iltisiana}
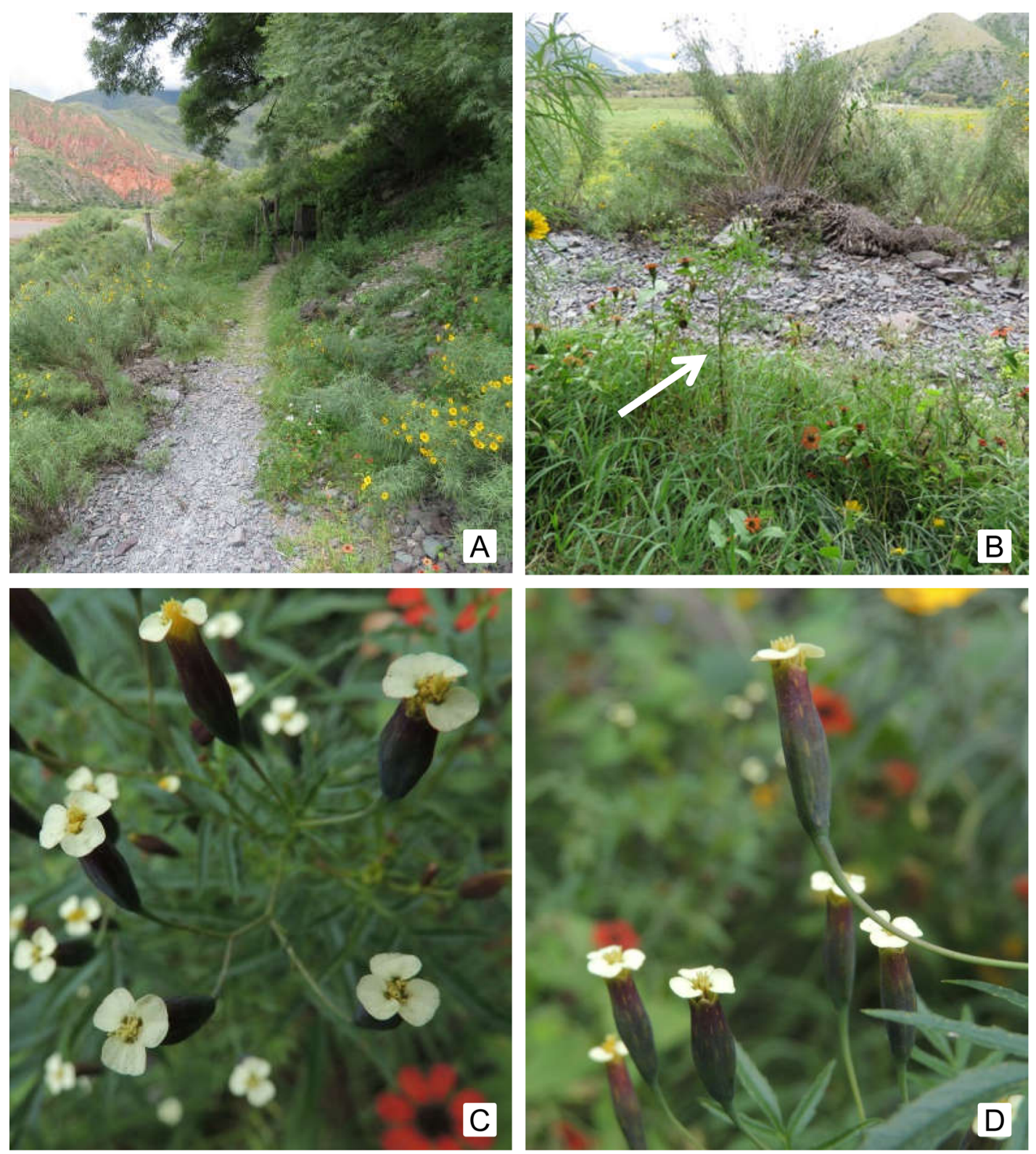

Fig. 2. Tagetes iltisiana. A: Hábitat. B: Planta (señalada por la flecha). C-D: Capítulos.

(Kenneth Cameron, com. pers.). Por otro lado, el ejemplar Cárdenas 4792 citado en el protólogo como material adicional no puede ser considerado material original de T. iltisiana, ya que el propio autor del nombre lo excluye explícitamente de la circunscripción de dicho taxón, debido a las diferencias encontradas en cuanto a la morfología del involucro y papus.
La información incompleta que proporciona el holotipo de T. iltisiana ha causado que numerosos especímenes coleccionados en Bolivia y Argentina no hayan podido ser identificados bajo este nombre, sino que fueron erróneamente determinados como otras especies de Tagetes con hábito similar (e.g. T. laxa, T. minuta, T. terniflora). De hecho, el ejemplar Burkart \& Troncoso 11569, citado 
en este trabajo como T. iltisiana, fue designado paratipo de T. cabrerae M.Ferraro (1955: 38), nombre incluido actualmente en la sinonimia de T. terniflora (Ariza Espinar 1967, Gutiérrez \& Stampacchio 2015). Posteriormente, el mismo ejemplar fue incluido en la exsiccata de T. laxa por Cabrera (1978), lo cual probablemente haya llevado a dicho autor a considerar en ese trabajo a esta última como una especie polimórfica, variable en el tamaño de las plantas y sus hojas.

Para la correcta aplicación del nombre, se designa un epitipo para T. iltisiana (Fig. 3), acorde al Código de Shenzhen, Art. 9 (Turland et al.. 2018). Este espécimen, depositado en BAA (BAA 00004840), fue coleccionado por el primer autor en la localidad jujeña de Volcán $\left(23^{\circ} 55,295^{\prime} \mathrm{S}\right.$ $\left.65^{\circ} 28,126^{\prime} \mathrm{W}\right)$. A partir de la designación de este especímen como tipo, se propone una descripción morfológica ampliada para T. iltisiana, que incluya caracteres que fueron omitidos en la descripción original, como también aquellos incorrectos que resulta necesario enmendar.

\section{Tratamiento taxonómico}

Tagetes iltisiana H.Rob., Phytologia 26: 378. 1973. TIPO: BOLIVIA. Dpto. Cochabamba: Prov. Cercado, Cervecería Colón, $2 \mathrm{~km} \mathrm{~N}$ de Cochabamba, $2700 \mathrm{~m}, 3-\mathrm{IV}-1963$, D. Ugent 4598 (Holotipo, US 00124917!). Epitipo (aquí designado!):-ARGENTINA. Prov. Jujuy: Dpto. Tumbaya, Volcán, $2105 \mathrm{~m}, 23^{\circ} 55^{\prime} 21,2^{\prime \prime} \mathrm{S}, 65^{\circ}$ 28'18,0" W, 13-III-2019, D. Schiavinato \& $M$. Anzuinelli 15/2019 (BAA 00004840!, duplicados BA!, BAA!, BAB!, CORD!, LIL!, LP!, MCNS!, MERL!, SI!) (Fig. 3).

Plantas anuales, (10-)20-40(-50) $\mathrm{cm}$ de altura, aromáticas. Tallos erectos o declinados, desnudos en la base y ramificados en la parte superior, estriados, glabros. Hojas herbáceas, opuestas inferiormente, las superiores alternas, subsésiles o cortamente pecioladas, láminas (2-)5-9(12) $\times(1-) 3-4(-5) \mathrm{cm}$, elípticas, pinnatisectas, divididas en 9-19 lóbulos, lóbulos (5-)15$25(-30) \times(1-) 2-4(-8) \mathrm{mm}$, ovados, aserrados, glándulas pelúcidas ovado-circulares, ubicadas en los márgenes, epidermis abaxial verde claro, glabra o con tricomas cortos escasos, epidermis adaxial verde oscuro, glabra. Capítulos radiados, heterógamos, agrupados en inflorescencias corimbiformes terminales (excepcionalmente solitarios en ejemplares precoces), laxas, cuyas ramas terminan en 2-3 capítulos, pedúnculos de $1-3 \mathrm{~cm}$ de longitud. Involucros 8-14 × 3-4 mm, subfusiformes o cilíndricos, verde oscuros en la parte proximal y purpúreos en la distal. Filarios 3 (ocasionalmente 4), en una sola serie, connados en su totalidad excepto el ápice, oblongos, glabros, cubiertos de glándulas lineares de 1-1,75 mm de longitud, las superiores elíptico-circulares, de $0,25 \mathrm{~mm}$ diámetro, ápice de los filarios triangular, 0,75-2 × 1-3 mm, obtuso, pubescente en el margen, mútico o con un mucrón apical de ca. 0,25 $\mathrm{mm}$. Receptáculo plano o ligeramente convexo, desnudo. Flores marginales (1-)3, pistiladas, corolas blanco-amarillentas, tipo radio verdadero, tubos de 3-4,5 $\mathrm{mm}$ de longitud, pubescentes; limbo $1,8-2,0 \times 2,5-3,0 \mathrm{~mm}$, oblato, ápice indiviso o apenas 2-3-lobado, glabro; ramas estilares $0,4-0,8 \mathrm{~mm}$ de longitud, lineares, ápice obtuso, papiloso. Flores del disco 3-6, perfectas, corolas (3-)4,5-6,25 $\mathrm{mm}$ de longitud, tubulosas, amarillas, pubescentes, lóbulos 5, 0,50-1,25 mm de largo, pubescentes en el lado adaxial. Anteras $5,0,8-1 \times 0,2-0,3 \mathrm{~mm}$, redondeadas en la base, apéndice conectival $0,30-0,60 \times 0,15-0,20 \mathrm{~mm}$, triangular, ápice obtuso; collar anteral $0,4-0,6$ $\times \quad 0,10-0,15 \mathrm{~mm}$, cilíndrico o subfusiforme; ramas estilares $0,75-1 \mathrm{~mm}$ de longitud, lineares, apéndice apical ca. 0,25 $\mathrm{mm}$ de longitud, cónico, obtuso, papiloso. Aquenios $6-9 \times 0,75-1 \mathrm{~mm}$, subfusiformes, pubescentes en toda su superficie, negros en la madurez. Papus formado por (8)10(-11) elementos paleáceos desiguales en una sola serie, 1-4 aristiformes, 3-6 $\mathrm{mm}$ de longitud, subulados, con margen escabroso, y los restantes escamiformes, $0,75-2 \mathrm{~mm}$ de longitud, con ápice setoso o fimbriado (Fig. 4).

Hábitat y distribución. Tagetes iltisiana habita áreas montañosas en Bolivia (Departamentos Cochabamba y La Paz) y el noroeste de Argentina (Provincias Salta y Jujuy), en la provincia fitogeográfica Prepuna (Cabrera \& Willink, 1980). Crece sobre laderas húmedas y bordes de caminos, hallada hasta el momento entre 1600 y 3100 metros sobre el nivel del mar.

Fenología. El período de floración es entre febrero y abril. 
D. J. Schiavinato y A. Bartoli - Clarificación y Epitipificación de Tagetes iltisiana

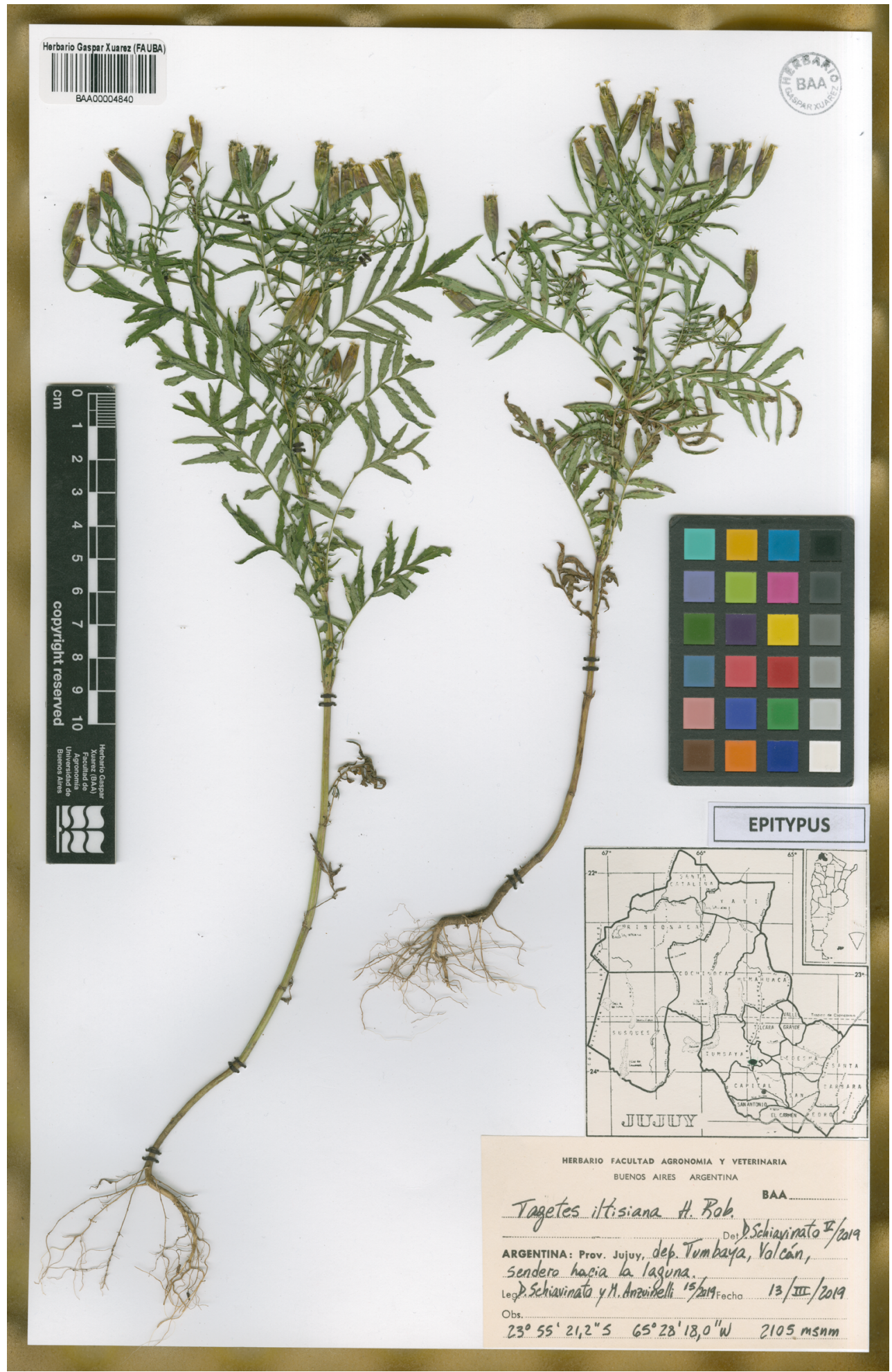

Fig. 3. Epitipo de Tagetes iltisiana (BAA). 
Bol. Soc. Argent. Bot. 54 (4) 2019
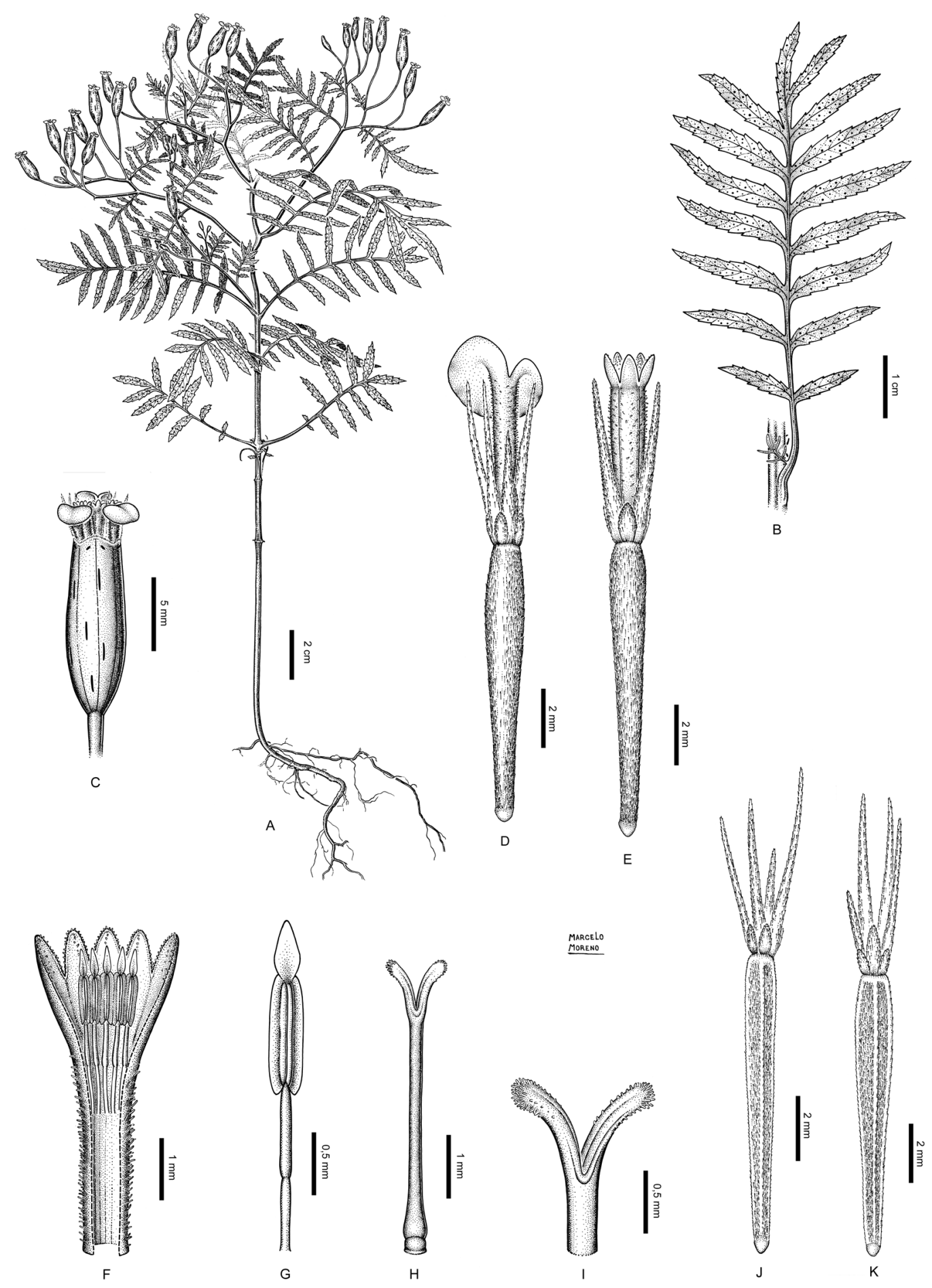

Fig. 4. Tagetes iltisiana. A: Planta. B: Hoja. C: Capítulo. D: Flor marginal. E: Flor del disco. F: Corola y androceo. G: Estambre. H: Estilo. I: Estigma. J-K: Aquenios. Escalas= A: $2 \mathrm{~cm}$; B: $1 \mathrm{~cm}$; C: $5 \mathrm{~mm}$; D-E: 2 $\mathrm{mm}$; F: $1 \mathrm{~mm}$; G: 0,5 mm: H: $1 \mathrm{~mm}$; I: 0,5 mm; J-K: $2 \mathrm{~mm}$. Dibujado por M. Moreno (D. Schiavinato \& M. Anzuinelli 15/2019, BAA00004840). 


\section{J. Schiavinato y A. Bartoli - Clarificación y Epitipificación de Tagetes iltisiana}

Etimología. El epíteto específico iltisiana es en honor al Dr. Hugh Iltis (1925-2016), profesor de Botánica y director del Herbario de la Universidad de Wisconsin (Robinson, 1973; Cochrane, 2017).

\section{Ejemplares adicionales examinados \\ Tagetes iltisiana}

ARGENTINA. Prov. Jujuy: Dpto. Valle Grande, Santa Ana, 3100 m, 28-II-1940, A. Burkart \& N. Troncoso 11569 (SI 017188). Prov. Salta: Dpto. Rosario de Lerma, Campo Quijano, 14-III-1945, D. Abbiatti \& L. Claps 478 (LIL 158783), Viaducto El Toro, 1600 m, 10-IV-1998, A. de Del Castillo 1178 (MCNS 004323). BOLIVIA. Dpto. Cochabamba: Prov. Cercado, Cochabamba, 2550 m, III-1939, P. Musch 78 (SI 131716). Dpto. La Paz: Prov. Larecaja, debajo de Sorata, en camino a San Pedro, punto estrecho del valle de San Cristóbal en la boca del río Ilabaya, a $20 \mathrm{~m}$ del río, $2540 \mathrm{~m}, 14-\mathrm{IV}-1985$, T. Feuerer 22302a (US 01808755).

\section{Tagetes laxa}

ARGENTINA. Prov. Salta: Dpto. Chicoana, Cuesta del Obispo, Quebrada de Lapacheta, 2880 m, 19-III-1972, A. Krapovickas et al. 22043 (BAA, LP, US), Ruta Provincial $33 \mathrm{~km} \mathrm{50,} \mathrm{a} 8 \mathrm{~km}$ al W de El Maray, 2250 m, 21-III-1997, J. R. De la Fuente et al. 10888 (CORD), Ruta Provincial 33 km 50, 2783 $\mathrm{m}, 25^{\circ} 10,261^{\prime} \mathrm{S} 65^{\circ} 49,751^{\prime} \mathrm{W}, 12-\mathrm{III}-2019, D$. Schiavinato \& M. Anzuinelli 12/2019 (BAA). Prov. Tucumán: Dpto. Tafí del Valle, Ruta Provincial 307, El Infiernillo, 2919 m, 26 43,035'S 65 47,595'W, 18-II-2016, D. Schiavinato et al. 63/2016 (BAA).

\section{Clave para las especies anuales de Tagetes que habitan Bolivia y el Noroeste Argentino}

1. Filarios truncados o levemente truncados, con ápice dentado. Glándulas del involucro punctiformes. Plantas con olor a anís. .. T. filifolia

- Filarios obtusos o agudos, múticos. Glándulas del involucro lineares, las superiores frecuentemente elípticas. Plantas con olor diferente al anís. ..... 2

2. Capitulescencias formadas siempre por capítulos multifloros y unifloros. T. terniflora

- Capitulescencias formadas únicamente por capítulos multifloros.

3. Papus laciniado, formado por elementos paleáceos con ápice dividido en 3-5 aristas (algunos elementos pueden ser 1-aristados).

T. praetermissa

- Papus no laciniado, formado por elementos paleáceos con ápice subulado o truncado.

4. Elementos paleáceos barbelados, con proyecciones laterales que igualan o superan al ancho de los mismos. ...................... T. multiflora

-Elementos paleáceos escabrosos, con proyecciones laterales mucho más cortas que el ancho de los mismos.

.. 5

5. Capitulescencias densas, con pedúnculos menores a $0,5 \mathrm{~cm}$ de largo. Involucros verdosos, raramente purpúreos. T. minuta

- Capítulos solitarios o en capitulescencias laxas, con pedúnculos mayores a $0,5 \mathrm{~cm}$ de largo. Involucros purpúreos, al menos en su tercio superior.

.. 6

6. Filarios 3(-4). Hojas discoloras, más claras en su cara abaxial. T. iltisiana

- Filarios 5-7. Hojas concoloras. 7

7. Flores marginales con limbo de la corola oblongoorbicular, con los márgenes no superpuestos con los de las flores marginales adyacentes. ... T. laxa

- Flores marginales con limbo de la corola oblato, con los márgenes superpuestos con los de las flores marginales adyacentes. T. imbricata

\section{Contribución DE los AUtores}

DS coleccionó el material de campo. Ambos autores realizaron un análisis exhaustivo de la información obtenida y contribuyeron de igual forma a la construcción de la descripción y la clave taxonómica. DS realizó el estudio nomenclatural y la epitificación de Tagetes iltisiana, con la ayuda de AB. DS escribió el manuscrito inicial y $\mathrm{AB}$ lo revisó para arribar a la versión final. Las fotografías fueron tomadas por DS y las figuras fueron elaboradas por $\mathrm{AB}$.

\section{Agradecimientos}

Agradecemos a las curadoras Gloria Barboza (CORD), Nora Muruaga (LIL), Laura Iharlegui (LP), Olga Martínez (MCNS), Norma Deginani y Amalia Suárez (SI) y Vicki Funk (US) por facilitarnos el acceso a las colecciones, al artista Marcelo Moreno por la ilustración del epitipo de Tagetes iltisiana, 
y a los revisores anónimos por las correcciones al manuscrito. Agradecemos especialmente a Milagros Anzuinelli por su invaluable colaboración durante el viaje de colección. Este trabajo fue realizado con el financiamiento de la Universidad de Buenos Aires y el Consejo Nacional de Investigaciones Científicas y Técnicas.

\section{Bibliografía}

ARIZA ESPINAR, L. 1997. Tagetes L. En: HUNZIKER, A. T. (ed.) Flora Fanerogámica Argentina 45, pp. 24-30. Museo Botánico de Córdoba, Córdoba.

CABRERA, A. L. 1937. Compuestas Argentinas nuevas o interesantes. Notas Mus. La Plata, Bot. 2: 171-204+ Figuras I-IV.

CABRERA, A. L. 1978. Compositae. En: CABRERA, A. L. (ed.) Flora de la Provincia de Jujuy. Colecc. Ci. Inst. Nac. Tecnol. Agropecu. 13: 1-726.

CABRERA, A. L. \& A. WILLINK. 1980. Biogeografia de América Latina. Washington D.C.: O. E. A. Serie de Biología, Monografía 13.

COCHRANE, T. 2017. Hugh Hellmut Iltis (1925-2016). Taxon 66: 527-532.

FERRARO, M. 1955. Las especies de Tagetes de la República Argentina. Bol. Soc. Argent. Bot. 6: 30-39.

GUTIÉRREZ, D. G. \& M. L. STAMPACCHIO. 2015. Tagetes. En: ZULOAGA, F. O., M. J. BELGRANO \& A. M. ANTON (eds.) Flora Argentina: Flora Vascular de la República Argentina, vol. 7 (2), pp. 118-129. Estudio Sigma SRL, Buenos Aires.

HIND, D. J. N. 2011. An annotated preliminary checklist of the Compositae of Bolivia 2. Disponible en: http:// www.kew.org/ (acceso 2 de mayo de 2019).

HUMBOLDT, F. W. H. A., A. J. A. BONPLAND \& C. S. KUNTH. 1818. Nova genera et species plantarum quas in peregrinatione ad plagam aequinoctialem orbis novi collegerunt, descripserunt, partim adumbraverunt (folio ed.), Vol. 4. Apud N. Maze, Bibliopolam, Paris.

LAGASCA Y SEGURA, M. 1816. Genera et species plantarum, quae aut novae sunt aut nondum recte cognoscuntur. Madrid.

LINNAEUS, C. VON. 1753. Species Plantarum 2. L. Salvius, Stockholm.

NEHER, R. T. 1966. Monograph of the genus Tagetes. Ph.D. thesis, Indiana University, Bloomington, Indiana.

ROBINSON, H. 1973. Additions to the genus Tagetes (Helenieae, Asteraceae). Phytologia 26: 378-380.
ROBINSON, H. 2014. Tagetes. En: JØRGENSEN, P. M., M. H. NEE \& S. G. BECK (eds.) Catálogo de las plantas vasculares de Bolivia. Monogr. Syst. Bot. Missouri Bot. Gard. 127: 290-382.

SCHIAVINATO, D. J. \& A. BARTOLI. 2018. Una nueva cita para la flora argentina: Tagetes praetermissa (Asteraceae, Tageteae). Bol. Soc. Argent. Bot. 53: 465-468. http://dx.doi.org/10.31055/1851.2372.v53.n3.21319

SCHIAVINATO, D. J. \& A. BARTOLI. 2019. Tagetes imbricata (Asteraceae, Tageteae), a new species from northwestern Argentina and Bolivia. Phytotaxa 408: 276-282.

https://doi.org/10.11646/phytotaxa.408.4.4

SCHIAVINATO, D. J., D. G. GUTIÉRREZ \& A. BARTOLI. 2017. Typifications and nomenclatural clarifications in South American Tagetes (Asteraceae, Tageteae). Phytotaxa 326: 175-188.

https://doi.org/10.11646/phytotaxa.326.3.2

SOULE, J. A. 1993. Systematics of Tagetes. Ph.D. thesis. University of Texas. Austin.

STROTHER, J. L. 1968. Vilobia, a new Genus (CompositaeTageteae). Brittonia 20: 343-345.

THIERS, B. 2019 [continuously updated]. Index Herbariorum: A global directory of public herbaria and associated staff. New York Botanical Garden's Virtual Herbarium [online]. Disponible en: http://sweetgum. nybg.org/science/ih/ (acceso 22 de marzo de 2019).

TURLAND, N. J., J. H. WIERSEMA, F. R. BARRIE, W. GREUTER, D. L. HAWKSWORTH, P. S. HERENDEEN, S. KNAPP, W. H. KUSBER, D. Z. LI, K. MARHOLD, T. W. MAY, J. MCNEILL, A. M. MONRO, J. PRADO, M. J. PRICE \& G. F. SMITH (eds.) 2018. International Code of Nomenclature for algae, fungi, and plants (Shenzhen Code) adopted by the Nineteenth International Botanical Congress Shenzhen, China, July 2017. [Regnum Veg. 159] Koeltz Botanical Books, Glashütten. https://doi.org/10.12705/Code.2018

ULlOA ULlOA, C., P. ACEVEDO-RODRÍGUEZ, S. BECK, M. J. BELGRANO, R. BERNAL, P. E. BERRY, L. BRAKO, M. CELIS, G. DAVIDSE, R. C. FORZZA, S. R. GRADSTEIN, O. HOKCHE, B. LEÓN, S. LEÓN-YÁNEZ, R. E. MAGILL, D. A. NEILL, M. NEE, P. H. RAVEN, H. STIMMEL, M. T. STRONG, J. L. VILLASEÑOR, J. L. ZARUCCHI, F. O. ZULOAGA \& P. M. JØRGENSEN. 2017. An Integrated Assessment of the Vascular Plants Species of the Americas. Science 358: 1614-1617. https://doi.org/10.1126/science.aao0398 